\title{
Flebotomíneos (Diptera: Psychodidae) de um foco de leishmaniose tegumentar no Estado de Minas Gerais
}

\author{
Sandflies (Diptera: Psychodidae) in an outbreak of cutaneous \\ leishmaniasis in the State of Minas Gerais
}

\author{
Edelberto Santos Dias', João Carlos França-Silva², Jaime Costa da Silva ${ }^{3}$, Érika Michalsky Monteiro', \\ Kenia Maria de Paula ${ }^{1}$, Caroline Macedo Gonçalves ${ }^{1}$ e Ricardo Andrade Barata ${ }^{1,}$
}

\begin{abstract}
RESUMO
Na localidade do Brejo do Mutambal, situado no município de Varzelândia (MG), área endêmica para leishmaniose tegumentar, foram realizadas capturas mensais sistemáticas utilizando-se armadilhas luminosas do tipo CDC durante o periodo de janeiro a dezembro de 2000. Foram capturadas 19 espécies de flebotomíneos, totalizando 6.756 exemplares. Lutzomyia intermedia (5,1\%), L. migonei $(0,4 \%)$ e L. whitmani $(0,1 \%)$, relacionadas com a transmissão de leishmaniose tegumentar, foram capturadas em número reduzido. Lutzomyia longipalpis foi a espécie predominante (34,8\%), sugerindo também um risco de transmissão da leishmaniose visceral. A proporção de insetos capturados no peridomícilio foi de 91,7\% enquanto no intradomicílio foi de $8,3 \%$. A interferência de fatores climáticos (temperatura, umidade relativa do ar e pluviosidade) sobre a dinâmica populacional de flebotomíneos foi avaliada.
\end{abstract}

Palavras-chaves: Leishmaniose tegumentar. Varzelândia. Minas Gerais. Flebotomíneos. Lutzomyia.

\begin{abstract}
Between January and December 2000, systematic monthly capture of phlebotomine sandflies was undertaken by means of light traps of CDC type in Brejo do Mutambal, an endemic area for American cutaneous leishmaniasis located in the municipality of Varzelândia, State of Minas Gerais. Nineteen phlebotomine species were captured, totaling 6,756 specimens. The species correlated with ACL transmission were captured in low numbers, as follows: Lutzomyia intermedia (5.1\%), L. migonei (0.4\%) and L. whitmani (0.1\%). Lutzomyia longipalpis was the predominant species captured (34.8\%), thus also suggesting a risk of visceral leishmaniasis transmission. The proportion of sandflies captured in areas surrounding bomes was $91.7 \%$ while $8.3 \%$ were captured inside homes. The interference of climatic factors (temperature, relative air humidity and rainfall) on phlebotomine population dynamics was evaluated.
\end{abstract}

Key-words: Cutaneous leishmaniasis. Varzelândia. Minas Gerais. Sandflies. Lutzomyia.

A leishmaniose tegumentar americana (ITA) é uma doença infecto-parasitária que acomete o homem sendo causada por várias espécies de protozoários do gênero Leishmania (Ross, 1903). Todas as espécies do gênero são transmitidas pela picada de fêmeas infectadas de dípteros da subfamília Phlebotominae, pertencentes aos gêneros Lutzomyia no Novo Mundo e Phlebotomus no Velho Mundo ${ }^{12}$.

No contexto da saúde pública, a LTA têm aumentado significativamente sua importância devido ao crescente processo de urbanização. Inicialmente, a doença tinha caráter eminentemente rural e, mais recentemente, vem se expandindo para áreas urbanas de médio e grande porte. Em franca expansão geográfica no Brasil, a LTA é considerada uma das infecções dermatológicas mais importantes, não só pela frequiência, mas principalmente pelas dificuldades terapêuticas, deformidades e seqüelas que pode acarretar.

As transformações no ambiente, provocadas pelo intenso processo migratório, o processo de urbanização crescente e

1. Centro de Pesquisas René Rachou, Fundação Oswaldo Cruz, Belo Horizonte, MG. 2. Universidade Federal de Minas Gerais, Belo Horizonte, MG. 3. Secretaria de Vigilância em Saúde, Belo Horizonte, MG.

Endereço para correspondência: Dr. Edelberto Santos Dias. Laboratório de Leishmanioses/CPqRR/FIOCRUZ. Av. Augusto de Lima 1715, Barro Preto, 30190-002 Belo Horizonte, MG, Brasil.

Fax $55313349-7758$

e-mail:edel@cpqrr.fiocruz.br

Recebido para publicação em 22/5/2006

Aceito em 12/1/2007 
as pressões sócioeconômicas acarretam a expansão das áreas endêmicas e o aparecimento de novos focos ${ }^{7}$. Desse modo, as espécies de flebotomíneos, que de alguma forma, resistem às condições adversas conseguem explorar novos ambientes, aproximando-se cada vez mais dos peridomicílios, facilitando a transmissão da doença ${ }^{1417}$.

O município de Varzelândia apresentou 303 casos humanos da doença no ano de 2000, sendo que 25 deles foram registrados na localidade do Brejo do Mutambal. 0 presente trabalho registra as espécies de flebotomíneos capturadas neste local, sua distribuição sazonal e seu comportamento em relação ao ambiente, associando à transmissão de ITA na área, visando estabelecer estratégias de controle passíveis de aplicação.

\section{MATERIAL E MÉTODOS}

O município de Varzelândia está localizado ao norte de Minas Gerais, no Polígono das Secas, com uma área territorial de $790 \mathrm{~km}^{2}$. Sua população consiste em 19.169 habitantes $^{10}$. 0 clima é tropical semi-úmido, com temperatura média de $24^{\circ} \mathrm{C}$, a estação seca prolonga-se por aproximadamente seis meses. O Brejo do Mutambal, área com alta prevalência de leishmaniose tegumentar americana foi selecionada para realização deste estudo.

Capturas sistemáticas foram realizadas em 9 residências, sendo uma armadilha exposta no intradomicílio e outra no peridomicílio, totalizando 18 armadilhas. Estas residências foram escolhidas previamente, levando-se em consideração o registro de casos humanos e 0 ambiente propício para criadouro, como a presença de plantações de banana e animais domésticos. Os flebotomíneos foram capturados com armadilhas luminosas do tipo $\mathrm{CDC}^{24}$, as quais foram expostas das 18:00 às 8:00h da manhã seguinte, durante quatro dias consecutivos por mês, no período de janeiro a dezembro de 2000.

Os espécimes capturados foram acondicionados em tubo de hemólise contendo álcool $70 \%$, sendo posteriormente levados ao Laboratório de Leishmanioses do Centro de Pesquisas René Rachou/FIOCRUZ. Em seguida, o material coletado foi previamente preparado e montado entre lâmina e lamínula, utilizando-se Bálsamo do Canadá para os machos e líquido de Berlese para as fêmeas ${ }^{11}$. Os espécimes foram identificados de acordo com a classificação proposta por Young \& Duncan ${ }^{25}$.

Os dados bioclimáticos de temperatura $\left({ }^{\circ} \mathrm{C}\right)$, umidade relativa do ar (\%) e pluviosidade $\left(\mathrm{mm}^{3}\right)$, referente ao período de estudo foram obtidos junto à Empresa de Pesquisas Agropecuárias de Minas Gerais (EPAMIG) sediado na Cidade de Janaúba (MG).

A interferência das variáveis climáticas (temperatura, umidade relativa do ar e pluviosidade) sobre o número de flebotomíneos capturados foi avaliada pelo teste de correlação de Pearson ${ }^{18}$ e quando as suposições de normalidade não foram satisfatórias foi usado o teste de correlação de Spearman através do software Prisma Versão 3.

\section{RESULTADOS}

No município de Varzelândia, na localidade do Brejo do Mutambal, durante o período de janeiro a dezembro de 2000, foram capturados 6.756 exemplares de flebotomíneos, sendo 3.894 machos e 2.862 fêmeas. Foram identificadas 19 espécies, sendo 18 do gênero Lutzomyia e um do gênero Brumptomyia, conforme Tabela 1.

Na Tabela 1 observamos o número total de flebotomíneos capturados com armadilhas luminosas tipo CDC, segundo sexo e ambiente, no Brejo do Mutambal. A proporção de insetos capturados no peridomícilio foi de $91,7 \%$ enquanto no intradomicílio foi de 8,3\%.

A distribuição relativa das espécies de flebotomíneos capturados no período compreendido entre janeiro e dezembro de 2000 está representada na Figura 1.

Tabela 1 - Flebotomíneos capturados com armadilba tipo CDC no Brejo do Mutambal, município de Varzelândia, segundo sexo e ambiente, janeiro a dezembro de 2000.

\begin{tabular}{|c|c|c|c|c|c|c|}
\hline \multirow{2}{*}{$\begin{array}{c}\text { Intradomicílio } \\
\text { Espécies }\end{array}$} & \multicolumn{2}{|c|}{ Peridomicílio } & \multicolumn{2}{|c|}{ Porcentagem } & \multicolumn{2}{|c|}{ Total } \\
\hline & $\hat{\sigma}$ & q & $\hat{\sigma}$ & q & $\mathrm{n}^{0}$ & $\%$ \\
\hline Brumptomyia avellari & 1 & 0 & 1 & 0 & 2 & 0,1 \\
\hline Lutzomyia capixaba & 0 & 0 & 1 & 3 & 4 & 0,1 \\
\hline L. cavernicola & 3 & 2 & 108 & 234 & 347 & 5,1 \\
\hline L. evandro $i$ & 2 & 3 & 16 & 2 & 23 & 0,3 \\
\hline L. intermedia & 95 & 20 & 158 & 70 & 343 & 5,1 \\
\hline L. ischnacantha & 9 & 1 & 218 & 49 & 277 & 4,1 \\
\hline L. lenti & 10 & 5 & 69 & 59 & 143 & 2,1 \\
\hline L. longipalpis & 81 & 51 & 1.813 & 412 & 2.357 & 34,8 \\
\hline L. migonei & 1 & 0 & 22 & 6 & 29 & 0,4 \\
\hline L. minasensis & 2 & 4 & 10 & 13 & 29 & 0,4 \\
\hline L. peresi & 13 & 13 & 25 & 17 & 68 & 1,0 \\
\hline L. quinquefer & 15 & 19 & 71 & 73 & 178 & 2,6 \\
\hline L. renei & 54 & 86 & 941 & 1.242 & 2.323 & 34,3 \\
\hline L. salles $i$ & 4 & 13 & 27 & 69 & 113 & 1,7 \\
\hline L. serrana & 0 & 0 & 2 & 1 & 3 & 0,1 \\
\hline L. shannoni & 1 & 0 & 5 & 0 & 6 & 0,1 \\
\hline L. sordellii & 0 & 0 & 1 & 1 & 2 & 0,1 \\
\hline L. trinidadensis & 3 & 0 & 4 & 0 & 7 & 0,1 \\
\hline L. whitmani & 2 & 0 & 2 & 0 & 4 & 0,1 \\
\hline Lutzomyia spp & 12 & 36 & 92 & 358 & 498 & 7,4 \\
\hline Total & 308 & 253 & 3.586 & 2.609 & 6.756 & 100,0 \\
\hline
\end{tabular}

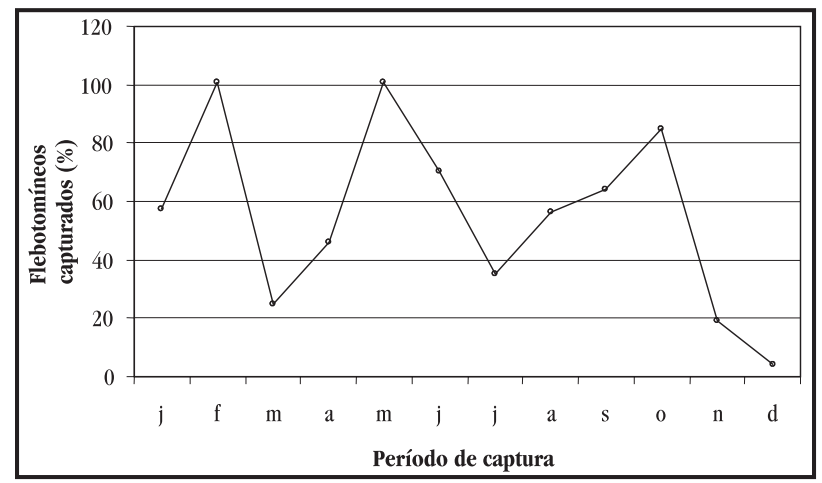

Figura 1 - Distribuição relativa do flebotomíneos capturados durante os o período compreendido entre janeiro e dezembro de 2000, Brejo do Mutambal, município de Varzelândia, Minas Gerais. 
A associação entre o número de flebotomíneos capturados, a variação da temperatura, umidade relativa do ar e pluviosidade está representada na Figura 2. Analisando o coeficiente de Pearson ou Spearman entre as variáveis independentes e a densidade dos flebotomíneos não se observou uma correlação positiva entre estas variáveis.

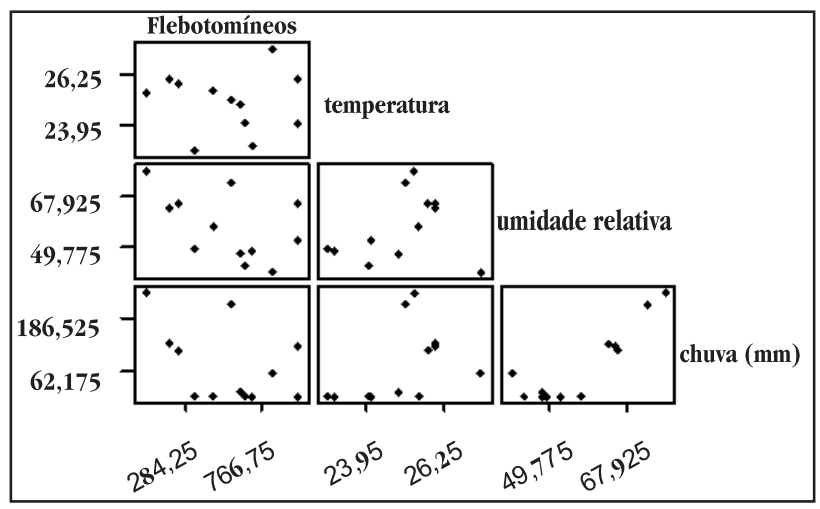

Figura 2 - Correlação entre a densidade populacional de flebotomíneos $e$ as variáveis independentes (temperatura, umidade relativa do ar e pluviosidade). Brejo do Mutambal, município de Varzelândia, MG no período compreendido entre janeiro a dezembro de 2000.

\section{DISCUSSÃO}

A fauna flebotomínica capturada na área do Brejo do Mutambal, município de Varzelândia, foi muito diversificada com 19 espécies identificadas, das quais $L$. longipalpis Lutz \& Neiva, 1912 e L. renei Martins, Falcão \& Silva, 1957 foram as espécies predominantes, representando, respectivamente, $34,8 \%$ e 34,3\% do total das espécies coletadas. Estes flebotomíneos caracterizamse por abrigarem-se em florestas primárias, secundárias e em rochas calcárias, preferencialmente. A adaptação que algumas espécies vêm sofrendo, principalmente em locais que sofreram ação humana, aproxima os vetores dos domicílios podendo facilitar a transmissão da $\mathrm{LTA}^{2} 3$.

Em estudos sobre o processo de urbanização de espécies de flebotomíneos e a transmissão das leishmanioses pôde-se encontrar espécies vetoras em áreas alteradas, como plantações de monoculturas, mas também no peridomicílio e intradomicílio. Neste caso, a LTA está associada à existência de vetores que são encontrados peridomiciliados, como a L. intermedia Lutz \& Neiva, 1912 e L. whitmani Antunes \& Coutinho, $1939^{8917}$. A existência de ambiente favorável à reprodução de flebotomíneos associado à presença de animais domésticos pode representar fatores que explicam o grande número de insetos nesse ambiente.

A espécie que apresentou maior densidade populacional durante o período de estudo foi $L$. longipalpis, sendo também assinalada em várias áreas endêmicas de leishmaniose visceral no Brasil. Sua alta capacidade vetorial associada à antropofilia $\mathrm{e}$ ao encontro desse inseto no intra e peridomicílio fazem, dessa espécie, o flebotomíneo de maior importância médica ${ }^{1623}$.

A ocorrência dos flebótomos em algumas áreas pode também estar relacionada com alguns fatores climáticos como temperatura, pluviosidade e umidade relativa ${ }^{15}{ }^{192}$. De acordo com essa hipótese, a chuva beneficia os flebotomíneos quando em níveis moderados ao longo da estação chuvosa, mas os prejudica quando inunda o chão, destruindo os criadouros e matando as pupas no solo ${ }^{20}$. Os habitats naturais dos flebotomíneos caracterizam-se por possuírem uma pequena variação na temperatura e umidade, o que favorece a presença desses insetos, já que os mesmos são muito sensíveis à dessecação. Uma pequena variação destes fatores nos microhabitats é suficiente para alterar a dinâmica das populações de flebotomíneos.

De acordo com a literatura, os fatores climáticos como temperatura, umidade e pluviosidade, influenciam de modo variável a população de flebótomos, dependendo da região analisada. Rutledge \& Ellenwood ${ }^{20}$ sugerem que a sazonalidade dos flebotomíneos está relacionada com os padrões de distribuição das chuvas que agem modificando as condições dos criadouros no solo. Comumente, estes insetos são encontrados em altas densidades durante os meses quentes e úmidos ${ }^{1921}$ ou em meses mais secos, como observado por Zeledon cols ${ }^{26} \mathrm{em}$ área da Costa Rica. No entanto, observou-se neste trabalho que estes fatores não constituíram fatores determinantes da densidade dos adultos, assim como encontrado por Condino e cols 5 .

Barata e cols ${ }^{4}$ mostraram a interferência de fatores climáticos sobre a densidade populacional de flebotomíneos no município de Porteirinha (Minas Gerais, Brasil), indicando uma correlação significativa entre o número de flebotomíneos capturados e a pluviosidade e umidade, enquanto que a temperatura não teve efeito significativo sobre a dinâmica destes insetos na região.

Embora se reconheça que o estudo de sazonalidade só apresenta dados confiáveis e consistentes quando se tem uma investigação de, no mínimo, dois anos consecutivos, para comparação dos resultados, diminuindo-se a margem de erros proporcionados por anos atípicos, neste estudo observou-se redução de densidade de flebótomos nos meses frios e secos, provavelmente devido às condições ambientais desfavoráveis para as formas imaturas.

De um modo geral, as residências da localidade do Brejo do Mutambal, situam-se muito próximas das formações de rochas calcárias, criadouros conhecidos destes insetos, aumentando as chances do contato homem/vetor podendo facilitar a transmissão de Leishmania. Este fato justifica o encontro do grande número de flebótomos capturados no peridomícilio (Tabela 1).

A maior proporção de machos, principalmente de L. longipalpis nas capturas com armadilhas do tipo $\mathrm{CDC}$, pode ser explicada como um comportamento natural dos machos em acompanhar as fêmeas para assegurar a fecundação durante seus deslocamentos. Este fato também foi observado em L. intermedia por Domingos e cols ${ }^{6}$.

Apesar do elevado número de L. longipalpis, na área não se observa uma relação proporcional ao número de casos de leishmaniose visceral. Provavelmente, isso se deve às medidas de controle aplicadas no local, como a eliminação de cães positivos, além de uma rigorosa educação com a comunidade e vigilância epidemiológica.

A presença de L. intermedia, L. migonei e L. whitmani, vetores comprovados de leishmaniose tegumentar, associado ao 
aumento significativo do número de casos da doença na área do Brejo do Mutambal, além de estudos da fauna flebotomínica e de comportamento de algumas espécies são de grande relevância epidemiológica, pois podem fornecer dados que irão subsidiar a escolha, pelos órgãos competentes, dos métodos de controle da doença mais adequados à situação atual.

\section{REFERÊNCIAS}

1. Aguiar AM, Soucasaux T. Aspectos da ecologia dos flebótomos do Parque Nacional da Serra dos Órgãos, Rio de Janeiro. I- Frequência mensal em isca humana (Diptera, Psychodidae, Phlebotominae). Memórias do Instituto Oswaldo Cruz 79: 179-209, 1984.

2. Aguiar GM, Vilela ML, Schuback PA, Soucasaux T, Azevedo ACR. Aspectos da ecologia dos flebótomos do Parque Nacional da Serra dos Órgãos, Rio de Janeiro. IV- Frequência mensal em armadilhas luminosas (Diptera, Psychodidae, Phlebotominae). Memórias do Instituto Oswaldo Cruz 80: 465-82, 1985.

3. Andrade-Filho JD, Carneiro APS, Lima MLN, Santiago RM, Gama MA, Santos CA, Falcão AL, Brazil RP. Flebotomíneos de Timóteo, Estado de Minas Gerais, Brasil (Diptera: Psychodidae). Cadernos de Saúde Pública 13: 767-770, 1997.

4. Barata RA, França-Silva JC, Fortes-Dias CL, Costa RT, Silva JC, Vieira EP, Prata A, Michalsky EM, Dias ES. Phlebotomines sand flies in Porteirinha, an endemic area of American visceral leishmaniasis in the State of Minas Gerais, Brazil. Memórias do Instituto Oswaldo Cruz 99: 481-487, 2004.

5. Condino MLF, Sampaio SMP, Henriques LF, Galati EAB, Wanderley DMV, Corrêa FMA. Leishmaniose tegumentar americana: flebotomíneos de área de transmissão no município de Teodoro Sampaio, região sudoeste do Estado de São Paulo, Brasil. Revista da Sociedade Brasileira de Medicina Tropical 31: 355-360, 1998

6. Domingos MF, Carreri-Bruno GC, Ciaravolo RMC, Galati EAB, Wanderley DMV, Corrêa FMA. Leishmaniose tegumentar americana: flebotomíneos de área de transmissão, no município de Pedro de Toledo, região sul do estado de São Paulo, Brasil. Revista da Sociedade Brasileira de Medicina Tropical 31: 425-32, 1998.

7. Fundação Nacional de Saúde. Manual de Controle da Leishmaniose Tegumentar Americana. $5^{\text {a }}$ edição. Ministério da Saúde, Brasília, 2000.

8. Gomes AC, Galati EAB. Aspectos ecológicos da leishmaniose tegumentar americana. 5. Estratificação da atividade espacial e estacional de Phlebotominae (Diptera, Psychodidae) em áreas de cultura agrícola da região de Vale do Ribeira, Estado de São Paulo, Brasil. Revista do Instituto de Medicina Tropical 82: 467$473,1987$.

9. Gomes AC, Rabello EX, Santos JLF, Galati EAB. Aspectos ecológicos da leishmaniose tegumentar americana. 1- Estudo experimental da frequência de flebotomíneos em ecótopos artificiais com referência especial a Ps. intermedius. Revista de Saúde Pública 14: 540-546, 1980.

10. Instituto Brasileiro de Geografia e Estatísticas. www.ibge.gov.br/cidadesat, 2003
11. Langeron M. Précis de Microscopie. Masson et Cie, Libraires de L'Académie de Medicine, Saint-Germain, Paris, 1949.

12. Killick-Kendrick R. The life-cycle of Leishmania in the sandfly with special references to the form infective to the vertebrate host. Annales de Parasitologie Humanie et Comparée 66: 71-74, 1991.

13. Killick-Kendrick R, Rioux JA. Intravectorial cycle of Leishmania in the sanflies. Annales de Parasitologie Humanie et Comparée 66: 71-74, 1991.

14. Marzochi MCA, Marzochi KBF. Tegumentary and visceral leishmaniases in Brazil. Emerging anthropozoonossis and possibilities for their control. Cadernos de Saúde Pública 10: 359-375, 1994.

15. Miscevic Z. Dependence of the flight of sandflies (Diptera, Phlebotomidae) in artificial light on the temperature and relative humidity. Acta Veterinary (Beograd) 31: 32-39, 1981.

16. Montoya-Lerna J, Cadena H, Oviedo M, Ready PD, Barazarte R, Travi BL, Lane RP. Comparative vectorial efficiency of Lutzomyia evansi and Lutzomyia longipalpis for transmitting Leishmania chagasi. Acta Tropica 85: 19-29, 2003.

17. Passos VM, Falcão AL, Marzochi MC, Gontijo CM, Dias ES, Barbosa-Santos EG, Guerra HL, Katz N. Epidemiological aspects of American cutaneous leishmaniasis in a periurban area of the metropolitan region of Belo Horizonte, Minas Gerais, Brazil. Memórias do Instituto Oswaldo Cruz 88: 103-110, 1993.

18. Pearson RD, Sousa AQ. Clinical spectrum of Leishmaniasis. Clinical Infectious Disease 22: 1-13, 1996.

19. Roberts DM. Arabian sandflies (Diptera, Psychodidae) prefer the hottest nights? Medical and Veterinary Entomology 8: 194-198, 1994.

20. Rutledge LC, Ellenwood DA. Production of plebotomine sandflies on the open forest floor in Panama: The Species. Environmental Entomology 4: 71-77, 1975.

21. Salomón OD, Rossi GC, Spinelli GR. Ecological aspects of phlebotomine ((Diptera, Psychodidae) in na endemic area of tegumentary leishmaniasis in the Northeastern Argentina, 1993-1998. Memórias do Instituto Oswaldo Cruz 97: 163-168, 2002.

22. Scorza JV, Ortiz I, Gomez I. Observaciones biologicas sobre algunos flebotomos de Rancho Grande (Venezuela). 6- Sobre los factores microclimáticos que determinan la endemicidad de la flebotomofauna de "Rancho Grande". Acta Biologica Venezuelica 6: 76-83, 1968.

23. Soares RPP, Turco SJ. Lutzomyia longipalpis (Diptera: Psychodidae: Phlebotominae): a review. Anais da Academia Brasileira de Ciências 75: 301-330, 2003.

24. Sudia WA, Chamberlain RW. Battery-Operated light trap: an improved model. Mosquitoes News 22: 126-129, 1962.

25. Young DG, Duncan MA. Guide to the identification and geographic distribution of Lutzomyia sand flies in Mexico, the West Indies, Central and South America (Diptera: Psychodidae). Memoirs of the American Entomological Institute 54: 1-881, 1994.

26. Zeledon R, Murrillo J, Gutierrez H. Observaciones sobre la ecologia de Lutzomyia longipalpis (Lutz \& Neiva, 1912) y posibilidades de existencia de leishmaniasis visceral en Costa Rica. Memórias do Instituto Oswaldo Cruz 79: 455-459, 1984. 